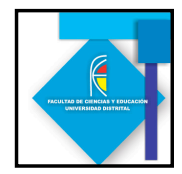

\title{
VERIFICACIÓN DEL EFECTO FOTO ELÉCTRICO CON LUZ ULTRAVIOLETA
}

\section{PHOTO ELECTRIC EFFECT CHECK WITH ULTRAVIOLET LIGHT}

\author{
A. L. Marquez ${ }^{1}$
}

\section{Resumen}

Desde el punto de vista de estudiante se desarrollo un experimento para verificar el efecto fotoeléctrico con un montaje sencillo para aclarar dudas sobre este efecto y las frecuencias de onda en las cuales se puede presentar, de igual manera se plantea la conexión matemática entre la ecuación de Einstein y la ecuación de trabajo en función de la carga del electrón para analizar la viabilidad de la cuantificación experimental de esta comprobación.

Palabras clave: Efecto Fotoeléctrico, Experimento.

\begin{abstract}
From the point of view of student development is an experiment to verify the photoelectric effect with a simple assembly to clarify questions concerning this effect and wave frequencies which can occur, just as there is the mathematical connection between the equation Einstein and the equation of work based on the electron charge to analyze the feasibility of experimental quantification of this check.
\end{abstract}

Keywords: Photoelectric Effect, Experiment

\section{Introducción}

En busca de nuevas estrategias para consolidar conceptos se puede crear un montaje sencillo para verificar el efecto fotoeléctrico y así todos los estudiantes de colegio y/o universidad puedan comprobar la base del funcionamiento de los paneles solares y aprovechar su auge.

${ }^{1}$ Fundación Universitaria Los Libertadores, angiemarquez80@hotmail.com 
$5^{\circ}$ CONGRESO NACIONAL DE ENSEÑANZA DE LA FÍSICA.

Universidad Pedagógica Nacional (U. P. N)

Universidad Distrital Francisco José de Caldas (U. D. F. J. C)

Bogotá, Colombia. 16 al 20 de mayo 2011

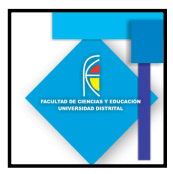

Al efectuar experimentos que comprueben el efecto fotoeléctrico generalmente se realizan con un equipo especial y con luz visible, por lo cual queda la duda en los practicantes si estos rangos también son validos para longitudes de onda diferentes a las de luz visible y se puede generar el mismo efecto.

El efecto fotoeléctrico es producto de paquetes de fotones provenientes de la luz (Tabla No. 1) proporcionan la energía suficiente para desprender o liberar electrones libres de un metal fotosensible. (Tabla No. 2).

\begin{tabular}{|c|c|c|c|}
\hline Color & $\begin{array}{c}\text { Rango } \\
\text { de } \\
\text { longitud } \\
\text { de onda } \\
\text { (nm.) }\end{array}$ & $\begin{array}{c}\text { Longitud de } \\
\text { onda } \\
\text { representativa }\end{array}$ & $\begin{array}{c}\text { Frecuencia } \\
\text { (hertzios) }\end{array}$ \\
\hline Ultravioleta & $<400$ & 254 & $11.8 \times 10^{14}$ \\
\hline Violeta & $400-425$ & 410 & $7.31 \times 10^{14}$ \\
\hline Azul & $425-490$ & 460 & $6.52 \times 10^{14}$ \\
\hline Verde & $490-560$ & 520 & $5.77 \times 10^{14}$ \\
\hline Amarillo & $560-585$ & 570 & $5.26 \times 10^{14}$ \\
\hline Anaranjado & $585-640$ & 620 & $4.84 \times 10^{14}$ \\
\hline Rojo & $640-740$ & 680 & $4.41 \times 10^{14}$ \\
\hline Infrarrojo & $>740$ & 1400 & $2.14 \times 10^{14}$ \\
\hline
\end{tabular}

Tabla No. 1 Rangos teóricos de longitud de onda de luz.

Para realizar este experimento se utilizo:

Recipiente de plástico.

Un par de placas de Aluminio, Bronce, Cobre y Zinc.

Fuente de luz ultravioleta.

Voltímetro.

Agua.

Se preparan las muestras mediante un proceso de oxidación y la otra debe estar libre de impurezas.

\begin{tabular}{|c|c|c|}
\hline Metal & $(\mathbf{e V})$ & $\begin{array}{c}\text { Frecuencia } \\
\text { (hertzios) }\end{array}$ \\
\hline Sodio & 2.46 & $5.95 \times 10^{14}$ \\
\hline Aluminio & 4.08 & $9.87 \times 10^{14}$ \\
\hline Cobre & 4.70 & $1.14 \times 10^{15}$ \\
\hline Zinc & 4.31 & $1.04 \times 10^{15}$ \\
\hline Plata & 4.76 & $1.15 \times 10^{15}$ \\
\hline Platino & 6.35 & $1.54 \times 10^{15}$ \\
\hline Plomo & 4.14 & $1.00 \times 10^{15}$ \\
\hline Hierro & 4.50 & $1.09 \times 10^{15}$ \\
\hline Estaño & 4.42 & $1.07 \times 10^{15}$ \\
\hline Bronce & 4.56 & $1.10 \times 10^{15}$ \\
\hline
\end{tabular}

Tabla No. 2 Función teórica de trabajo de algunos metales

*Como el bronce es aleación de algunos de estos metales, se considera la función de trabajo del mismo orden. 
Se perfora el recipiente de plástico para que la fuente de luz ultravioleta lo atraviese, de esta forma la fuente de luz quede sumergida en agua igual que las placas del mismo material siempre estando una oxidada y la otra libre de impurezas, las cuales estaban conectas al voltímetro para medir el flujo de electrones desprendidos (Ver Fig. 1).

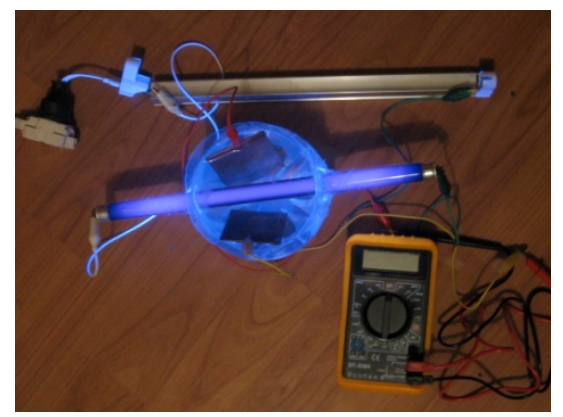

Fig. 1. Montaje del experimento para verificación del efecto fotoeléctrico.

\section{Resultados y Análisis.}

\begin{tabular}{|c|c|c|c|c|}
\hline $\begin{array}{c}\text { Material } \\
\text { Oxidado }\end{array}$ & $\begin{array}{c}\text { Material } \\
\text { Libre }\end{array}$ & $\begin{array}{c}\text { Voltaje } \\
\text { Inicial }\end{array}$ & $\begin{array}{c}\text { Voltaje } \\
\text { Final }\end{array}$ & $\begin{array}{c}\text { Diferencia } \\
\text { de } \\
\text { Voltaje } \\
(\boldsymbol{V})\end{array}$ \\
\hline Aluminio & Aluminio & 0.15 & 0.96 & 0.81 \\
\hline Bronce & Bronce & 0.41 & 0.72 & 0.31 \\
\hline Cobre & Cobre & 0.35 & 0.52 & 0.17 \\
\hline Zinc & Zinc & 0.73 & 1.31 & 0.58 \\
\hline
\end{tabular}

Tabla No. 3 Medición de Voltaje.

Al realizarse en aire las partículas se dispersan y no alcanza a registrarse ninguna medición en la placas, por ello se realiza en agua que contiene átomos o moléculas presentes en el medio que han ganado o perdido electrones de su dotación normal llamados iones con carga negativa y positiva facilitando el transporte de los electrones desprendidos por la placa por acción de la luz.

Estos iones libres registran un voltaje el cual se toma como voltaje inicial ya que al encender la fuente de luz ultravioleta se registra un cambio en la lectura del voltaje que varía dependiendo el material que se esté utilizando y que desciende al apagar la fuente. (Tabla N. 3).

La frecuencia de la luz ultravioleta en el agua es constante pero la longitud de onda varia en el agua, entonces:

$=$

Donde:

: Longitud de onda de la luz

: Velocidad de medio donde se propaga la luz 


\section{$5^{\circ}$ CONGRESO NACIONAL DE ENSEÑANZA DE LA FÍSICA. \\ Universidad Pedagógica Nacional (U. P. N) \\ Universidad Distrital Francisco José de Caldas (U. D. F. J. C) \\ Bogotá, Colombia. 16 al 20 de mayo 2011

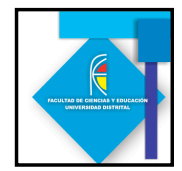

: Frecuencia de la longitud de onda de luz

: Velocidad en el agua es $2.25 \times 108 /$

$=2.25 \times 108 / 11.8 \times 1014$

$=1.91 \times 10-7$

$=191$

La longitud de onda de la luz ultravioleta en el agua es de $191 \mathrm{~nm}$.

Las ecuaciones relacionadas para comprobar este efecto son las siguientes:

$=h$. -

$$
=h .0
$$

$=h \cdot-\hbar \cdot 0$

$$
=h(-0)
$$

Donde

: Energía cinética máxima de un electrón emitido

$h$ : Constante de Plank $=6.626 \times 10-34$.

: Frecuencia de la longitud de onda de la luz

: Función trabajo necesaria para extraer un electrón de la superficie metálica

0 : Frecuencia umbral del metal

Aluminio - Aluminio

$$
\begin{aligned}
& =6.626 \times 10-34.11 .8 \times 1014 h-6.54 \times 10-19 \\
& =1.28 \times 10-19
\end{aligned}
$$

Bronce - Bronce

$$
\begin{aligned}
& =6.626 \times 10-34 \cdot 11.8 \times 1014 h-7.53 \times 10-19 \\
& =5.13 \times 10-20
\end{aligned}
$$

Cobre - Cobre

$$
\begin{aligned}
& =6.626 \times 10-34 \cdot 11.8 \times 1014 h-7.31 \times 10-19 \\
& =2.89 \times 10-20
\end{aligned}
$$

Zinc - Zinc

$$
\begin{aligned}
& =6.626 \times 10-34 \cdot 11.8 \times 1014 h-6.91 \times 10-19 \\
& =9.14 \times 10-20
\end{aligned}
$$

Para comparar el valor del voltaje obtenido en el experimento se necesita una conexión entre la energía cinética encontrada y el voltaje, para ello se utiliza la siguiente ecuación:

Despejando voltaje tenemos:

$=$

Donde

: Energía cinética máxima de un electrón emitido 
$5^{\circ}$ CONGRESO NACIONAL DE ENSEÑANZA DE LA FÍSICA.

Universidad Pedagógica Nacional (U.P.N)

Universidad Distrital Francisco José de Caldas (U. D. F. J. C)

Bogotá, Colombia. 16 al 20 de mayo 2011

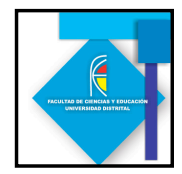

: Voltaje

: Carga del electrón $=1.6 \times 10-19$

Aluminio:

$=1.28 \times 10-191.6 \times 10-19$

$=0.8013$

Bronce:

$=5.13 \times 10-201.6 \times 10-19$

$=0.3207$

Cobre:

$=2.89 \times 10-201.6 \times 10-19$

$=0.1805$

Zinc:

$=9.14 \times 10-20 \quad 1.6 \times 10-19$

$=0.5710$

\begin{tabular}{|c|l|l|l|}
\hline Metal & & & Voltaje \\
\hline Aluminio & & & \\
\hline Bronce & & & \\
\hline Cobre & & & \\
\hline Zinc & & & \\
\hline
\end{tabular}

Tabla No. 4 Valores teóricos del experimento.

Con el valor del voltaje experimental se encontró la energía cinética de cada material y la función trabajo del mismo, así:

$$
=\quad \mathrm{y}=\hbar \cdot-
$$

Despejando, se tiene:

$=h$. -

Aluminio:

$$
\begin{aligned}
& =1.6 \times 10-19(0.81) \\
& =1.30 \times 10-19
\end{aligned}
$$

$=6.626 \times 10-34 \cdot 11.8 \times 1014 h-(1.30 \times 10-19)$

$=6.53 \times 10-19$

Bronce:

$$
\begin{aligned}
& =1.6 \times 10-19(0.31) \\
& =4.96 \times 10-20
\end{aligned}
$$

$=6.626 \times 10-34 \cdot 11.8 \times 1014 h-(4.96 \times 10-20)$ 


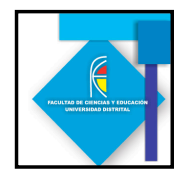

$$
\begin{aligned}
& =7.33 \times 10-19 \\
& \text { Cobre: } \\
& \quad=1.6 \times 10-19(0.17) \\
& \quad=2.72 \times 10-20 \\
& =6.626 \times 10-34.11 .8 \times 1014 h-(2.72 \times 10-20) \\
& =7.55 \times 10-19 \\
& \text { Zinc: } \\
& \quad=1.6 \times 10-19(0.58) \\
& \quad=9.28 \times 10-20 \\
& =6.626 \times 10-34.11 .8 \times 1014 h-(9.28 \times 10-20) \\
& =6.90 \times 10-19
\end{aligned}
$$

\begin{tabular}{|c|l|l|l|}
\hline Metal & & & Voltaje \\
\hline Aluminio & & & \\
\hline Bronce & & & \\
\hline Cobre & & & \\
\hline Zinc & & & \\
\hline
\end{tabular}

Tabla No. 5 Valores experimentales.

\section{Conclusiones}

Al comparar los valores teóricos con los valores experimentales presenta un margen de error inferior al $5 \%$, por lo cual se puede decir que el experimento es válido.

Para el desarrollo de este experimento no se necesitan costosos equipos, sus materiales son fáciles de conseguir y su montaje es sencillo (Fig. N. 1) por lo cual se puede realizar en cualquier laboratorio de universidad o colegio.

Se requiere realizar el montaje en agua porque en el aire los electrones desprendidos por la luz ultravioleta sobre las placas de metal son liberados pero no con la suficiente energía para desplazarlos, en el agua al haber cargas negativas y positivas (iones) posibilita el desplazamiento de los electrones.

Al analizar el efecto fotoeléctrico se requiere como complemento estudiar la energía en función de la carga de electrón para la viabilidad de la cuantificación del efecto fotoeléctrico por parte de los practicantes.

\section{Referencias Bibliográficas}

SERWEY, Raymond, Física para Ciencias e Ingeniería, Tomo II. 5ta Edición. México, Editorial Mc Graw Hill. 2002.

SADIKU, Matthew Elementos de Electromagnetismo. 2da Edición. México. Editorial Alfaomega. 2007. 


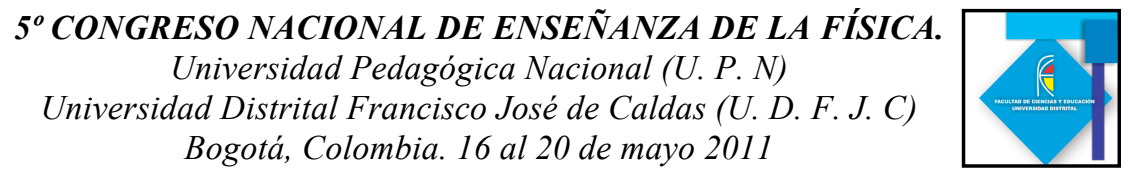

ECNIRAMA. Tomo VII. Buenos Aires, Argentina. Editorial Codex S.A.1963.

Grup d'Estudi de la Natura del Moianès [en linea] URL: http://www.joseplainez.org/GRUP\%20MOIANES/RECOPILACIO TECNICA/Naturaleza \%20de\%20la\%20luz.htm

Planeta Sedna [en línea] URL: http://www.portalplanetasedna.com.ar/fotoelectrico.htm 